\title{
Analysis and assessment of targets on a digital map and Computer processing of Intelligence data
}

\author{
FURJÁN Attila ${ }^{1}$
}

\begin{abstract}
The Tactical Operational Command and Control Information System shall have sub-systems that are capable of covering, ensuring, and servicing - in summary supporting - the battlefield systems operated by the combat (manoeuvre) forces and combat support units and sub-units during planning and commanding, on the land forces, brigade, battalion and company level.

One of the most important such sub-systems is the intelligence planning, command and data processing functional sub-system. It allows for a quick and professional processing of target intelligence data, displaying them with the standard symbols on digital maps, and promptly grouping them by priority; thereby it greatly facilitates the manoeuvre commander's foresight, provides assistance for decisionmaking, and provides accurate target data to the fire support groups for target planning.

Head words: "HUTOPCCIS" command system, target intelligence, target reporting, target analysis, target data processing, digital map, geospatial information systems, intelligence planning on digital maps, visibility examination, detected targets, monitoring of fire for effect.
\end{abstract}

\section{Intelligence data reporting and entering into the computer}

The collection and processing of intelligence data would be carried out by the intelligence data processing- and command post, which is to be established at the command posts of combat troops (brigade, land force); and by the intelligence data processing sections (FAR) to be established at the fire control posts of the artillery. Thus, the data necessary for fire control would be immediately available through the information network, and close cooperation would be established between the two groups.

The units directly carrying out reconnaissance, belonging to different intelligence organization types, report the intelligence data using a standard intelligence-reporting form, the "Intelligence Log", to the intelligence data processing- and command post (FAVP) and to the intelligence data processing section (FAR) via radio or a line telecommunications device if the means of entering digital data into the computer has not yet been provided (Fig. 6).

The recce sub-units would make reports in the "Intelligence Log" in the columns marked black. The numbering of these columns is in line with the computer data entry (Fig. 1-2).

1 National University of Public Service, Budapest, Hungary 


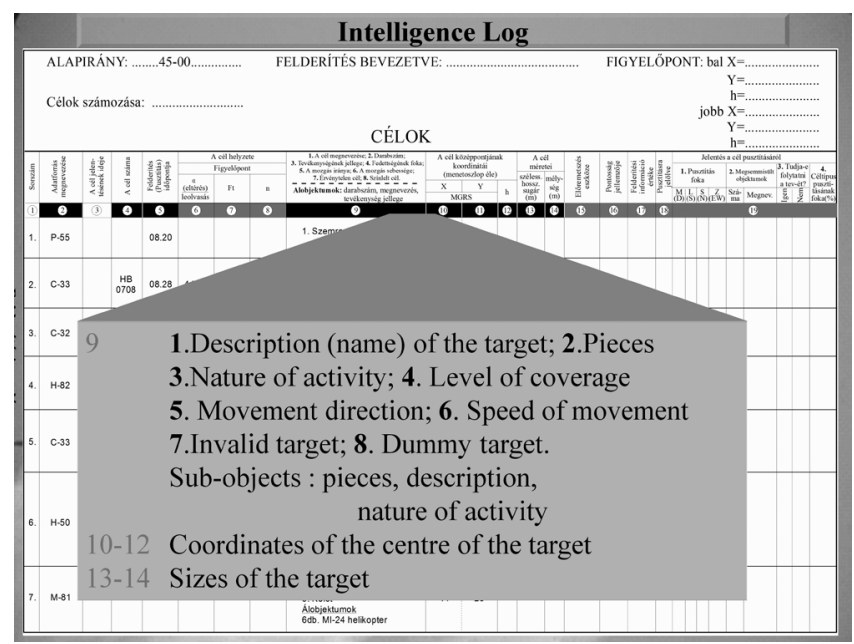

Figure 1. Standard intelligence log (own resources)

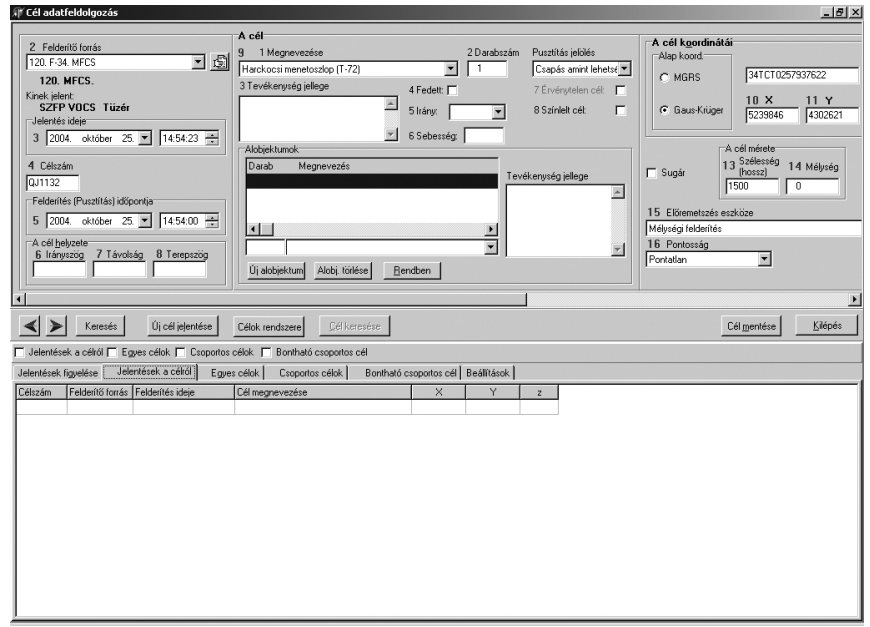

Figure 2. The developed digital log (own resource)

Polar coordinates (direction and distance) can also be reported, in which case columns 6, 7, and 8 shall be filled. For columns 15-18, only those reconnaissance units shall report which have no data in the data base.

The operator of the intelligence and data processing centre takes up the report via radio or a line device, and enters the data into the computer, following the order of the report.

The software automatically displays and records the time of report when the reported intelligence data are entered. In column 9 of the "Intelligence Log”, text input is also possible. All sub-objects of a grouped target can be entered and these targets will also be summarized by type and nature of target. Of course, besides the type of target, they can report here other important enemy information found in a visual way with map-terrain synchronization or estimation. Example: “Muhi populated settlement D-1500, enemy has activated infantry-, 
armour-piercing gun and mortar firing, probably preparing for an assault”. Such and similar reports can be made by border guards and different reconnaissance patrols, who inspect enemy forces visually, possibly using binoculars. These groups usually do not have any apparatuses, probably only maps, so they will specify the location of the target through estimations, in relation to an object or a survey point that can be identified on the map.

Further information can be reported about a previously indicated target (target no.), and the software will record the report for that same target with the actual time of reconnaissance.

For reporting in columns 10-11, coordinates can be entered using the UTM-system, consistent with NATO requirements (Military Grid Reference System - MGRS coordinates). If a target is reported with shorter coordinates, the software will take up the middle of the specified square as the location of the target.

For no. 16, the accuracy indicator of positioning by the intelligence data can be one of the following:

"P” — accurate, “Pn” — inaccurate.

Accuracy is a basic requirement for the specification of the coordinates, height above sea level, and size of detected targets; which ensures the efficiency of destruction by fire.

Accuracy requirements:

- the error of accuracy of specifying the coordinates of the targets shall not be more than 75 m (Hungarian artillery guidelines of Tü/1, Tü/50);

- the width and depth error of accuracy when specifying the size of groups of targets shall not be more than $100 \mathrm{~m}$ (Hungarian artillery guidelines of Tü/1, Tü/50);

- the error of accuracy of specifying the height of the targets shall not be more than $5 \mathrm{~m}$;

Accurate (“P”): if the above accuracy requirements are met.

Inaccurate ("Pn"): if the error of accuracy of specifying the coordinates of the targets is more than $75 \mathrm{~m}$.

Every reconnaissance asset or method has its characteristic accuracy of specifying coordinates, which is symbolized by the average error (Ek). As the average error is a component of the cumulative error of fire aiming, the result of firing directly depends on it. Therefore, the effect of this average error can be assessed through the probability of accomplishment of the firing mission.

Colonel S. Zajcev, Academy Counsellor, Csc (PhD) of Military Sciences, Colonel V. G. Ramicün, Csc (PhD) of Military Sciences, and Major A.N. Sautin conducted research about the dependence of the probability of the destruction of different targets on the average error of specifying their coordinates. (Chart 2). 


\begin{tabular}{|c|c|c|c|c|}
\hline \multirow[b]{2}{*}{ Description } & \multicolumn{2}{|c|}{ Sizes (m) } & \multirow{2}{*}{$\begin{array}{l}\text { Average error of } \\
\text { specifying } \\
\text { coordinates (m) }\end{array}$} & \multirow{2}{*}{$\begin{array}{l}\text { Probability of } \\
\text { accomplishment of } \\
\text { the firing mission }\end{array}$} \\
\hline & $\begin{array}{l}\text { width } \\
\text { (m) }\end{array}$ & $\begin{array}{l}\text { length } \\
\text { (m) }\end{array}$ & & \\
\hline $\begin{array}{l}\text { Firing line of a self- } \\
\text { propelled battery } \\
\text { incl. } 6 \text { guns situated } \\
\text { in firing position }\end{array}$ & 250 & 150 & $\begin{array}{c}30 \\
50 \\
70 \\
100\end{array}$ & $\begin{array}{l}0.82 \\
0.62 \\
0.39 \\
0.08\end{array}$ \\
\hline $\begin{array}{l}\text { Division Command } \\
\text { Post }\end{array}$ & 320 & 320 & $\begin{array}{c}30 \\
50 \\
70 \\
100\end{array}$ & $\begin{array}{l}0.96 \\
0.89 \\
0.76 \\
0.48\end{array}$ \\
\hline
\end{tabular}

Chart 1. Relation between the probability of accomplishing the firing mission and the average error of specifying their coordinates

The chart shows that with the increase of the average error of specifying coordinates (Ek), the possibility of accomplishing the firing mission decreases significantly, especially if the average error increases to or over $70 \mathrm{~m}$. The estimations made by scientists have shown that such dependence applies to other types of objects, too.

Column 17 of the "Intelligence Log" shall be filled and submitted in case the information value reported by the intelligence source differs from the data already included in the data base, and they can properly affirm that fact.

The value of information shall be indicated using the NATO standard system

Indications of reliability of the data source and the data collection organ:

- “A - completely reliable;

- B - usually reliable;

- C - sufficiently reliable;

- D - usually not reliable;

- E - not reliable;

- F - reliability cannot be assessed.”

"Rating 'A' occurs very rarely. This rating can be used if the source is known to have broad experience and background with regards to the reported type of information. Rating ' $B$ ' means an information source whose integrity is known. Rating ' $F$ ' means there is no basis for assessing the reliability of the source. Data collection organs are usually rated A, B, or C. ... if judgements about the data source and the reporting organ differ, the lower indication shall be applied." [1] The reliability of the intelligence source is collected from the reporting organ and entered into the data base.

The other component of assessing information value is accuracy, which is indicated by a number. The assessment shall be done by the lowest possible level. The classification of the information by accuracy for actual (real) targets is shown in Figure 8.

The accuracy indicators for data collection organs are also entered into the data base in the combat preparation phase. This shall be known by data collection and reporting organs, too. If the judgement regarding the value of information corresponds to the data included in 
the data base, no report shall be submitted in column 17 of the "Intelligence Log”. Otherwise the value of information shall be reported.

Information confirmed by other sources is defined as information established (detected) through several intelligence methods independently. These data will have rating " 1 ", and they shall be - beyond any doubt whatsoever - correspondent to the situation and to the location of the target.

The information is considered obviously correct if it is correspondent to the situation and to the expected enemy activity and has been established through only one intelligence method and on one occasion. Also in cases where the essential parts of the report are confirmed based on already available information. Information of such qualification will be rated " 2 ", and in this case additional intelligence will be needed.

"If a reported fact, regarding which no further information is available, is assessed as conforming to the previously observed activities of the target, then it will be qualified 'probably' correct and rated ' 3 '.”' [1: 28]

In this case, additional intelligence is necessary.

Unconfirmed information that does not conform to the observations regarding the activities of the target, will be qualified "highly doubtful" and rated " 4 ".

Information deemed "unlikely", that is not confirmed by the available data, is consistent with the actual situation but not with other data previously gathered from different sources and processed. The information which is inconsistent with already available data having a rating of " 1 " or " 2 "will have this qualification, too.

These data need clarification, additional intelligence, and confirmation by preferably more than one intelligence methods.

"Verity cannot be assessed" qualification applies to those intelligence data which have no evidences for a classification on the 1-5 assessment scale due to lack of data regarding the target. In this case, additional intelligence is necessary.

It must be noted that a fully reliable data collection organ may gather and report from fully reliable sources such data that will in turn be assessed as unlikely when compared to other information. Such information will be rated “A-5”. A source assessed as unreliable may also report information that proves to be accurate when compared to other sources. In that case, the value of the information is $\mathrm{E}-1$.

Information with rating "2-6” will be automatically appointed for additional intelligence.

All the intelligence information rated " $1-6$ " belongs to the group of actual (real) targets. (Fig. 3) 


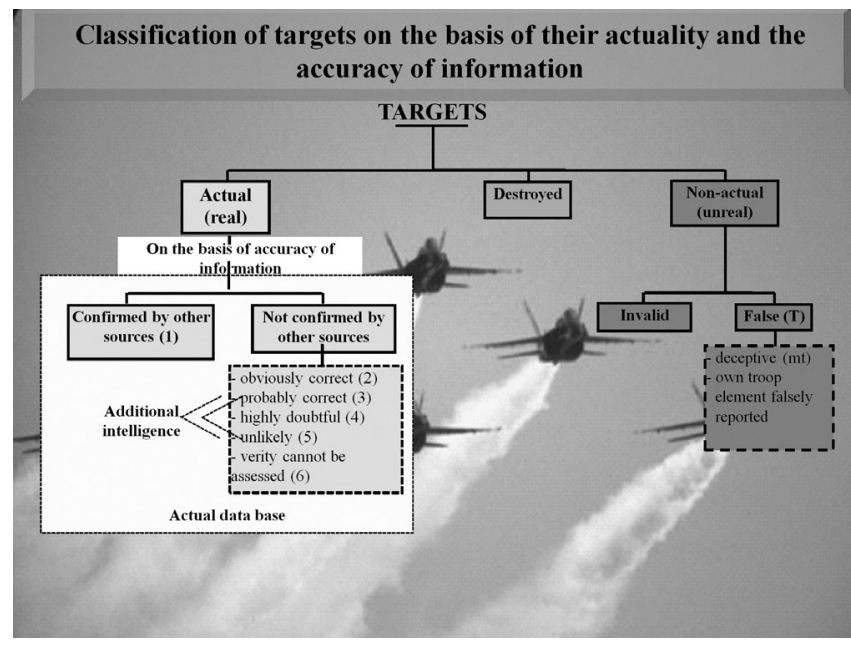

Figure 3. Classification of targets after processing, on the basis of their actuality and the accuracy of information (own resources)

However, some targets are deployed by the enemy in order to deceive our troops. These dummy targets, belonging to the group of false targets within the category of non-actual (unreal) targets, are all deceptive targets. (Fig. 3)

Deceptive intelligence data are those which do not conform with the actual situation, are inconsistent with data collected from other sources, and undoubtedly proven to be false. These data are taken into account with the aim of investigating the deception methods of the enemy and collecting intelligence about their misinformation system (Fig. 3).

"In the univocal opinion of foreign military experts, the extent to which the expected losses of the artillery sub-units can be decreased is practically equal to the number of dummy targets. If the amount of dummy firing positions is $20-30 \%$ of the amount of real ones, the losses will decrease by 32-34\%. This has been proved in the Persian Gulf War, too.” [2] Therefore, a thorough analysis and synthesis of all data shall be carried out during the analysis, assessment, and processing of intelligence data.

The reports on "targets" also belong to the group of false targets where the location of own troop elements has been specified by graphic intersection and reported by mistake.

If a target (information) loses actuality, which the intelligence source reports, then it will be invalidated and therefore moved from the actual data base to the group of invalid targets (Fig. 3).

Destroyed targets will also be taken out of the actual data base and moved to a separate group of targets. Both the destroyed and deceptive (dummy) targets have distinct symbols on the digital map.

Experience from intelligence exercises show that reported intelligence data rarely comply with the requirements of actuality and accuracy of specification of coordinates, and with the strict requirements of information assessment. Even intelligence data representing high information value and accuracy (of positioning) often do not ensure the timely accomplishment of firing missions, primarily due to the slow processing of intelligence data, and also reversely: the timely submitted reports may have low value due to the inaccuracy of coordinates. 
With the reporting process, data entry is finished and computer processing starts.

\section{Processing of intelligence data}

"Processing — the development of intelligence results through collection, definition of value, analysis, and integration of information and/or other intelligence data.” [3: 53]

Data processing is carried out on different levels, including the assessment done by the data collection organ, and up to the highest level. The lowest-level data processing is called pre-assessment, and it usually only involves the conversion of raw data into a comprehensible form (e.g. interpreting the symbols of the locator screen or editing patrol reports). On this level, the gathered data can still be compared to the data formerly collected by the data source.

Data processing actions include three parts:

"1. Recording: the depiction of the collected information in a written or other graphic form, and the allocation of pieces of information to the associated groups.

2. Defining the value: the clarification of the relation between the intelligence data and the operations, the reliability of the data source or data collection organ, and the accuracy of information.

3. Analysis: defining the importance of the information — in relation to the already known data and intelligence information — and drawing conclusions regarding the anticipated meaning of the assessed information." [1: 18]

Data processing is a continuous activity.

\section{Computer processing}

When a target is reported, it is instantly entered into the system of targets, that is, the target type, target subtype, nature of target, and group of targets will be specified. The software classifies the targets into four priority groups:

- Group I: High Pay-off Targets (HPTs);

- Group II: High Value Targets (HVTs);

- Group III: Important Other Targets;

- Group IV: Other Targets (Fig. 9). 


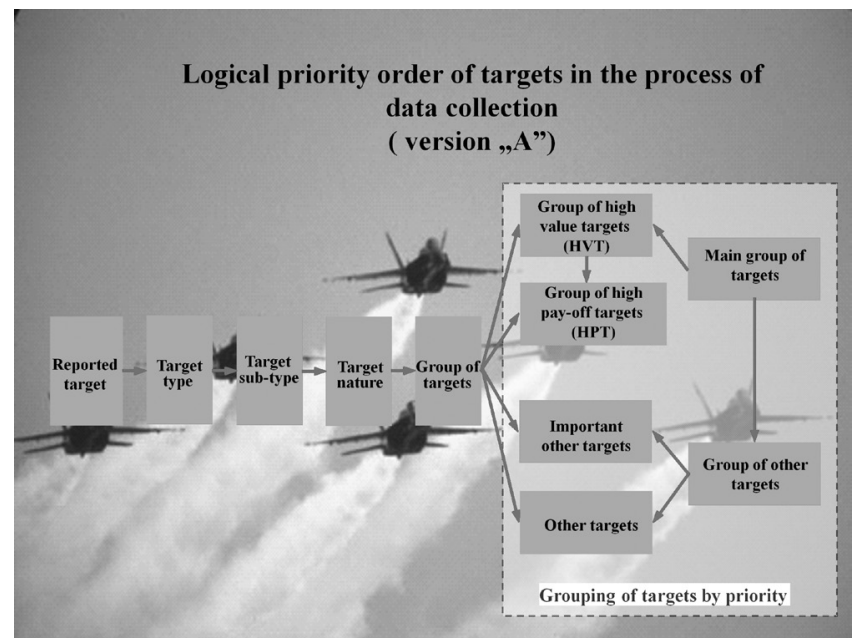

Figure 4. Computer processing of the reported targets (own resources)

The priority of intelligence data determines their value and is directly related to their urgency.

High Pay-off Targets are selected from High Value Targets. These targets can have essential (critical) impact on one's own troops in the course of the operation (combat), therefore, they must be destroyed immediately.

The present literature focuses on the high value targets and the high pay-off targets, and does not address the analysis of "other" targets. The group of high value targets is very broad. The armed forces of a small country have a limited number of intelligence assets and firing means of combat. In our research, we have come to the conclusion that the wide scale of other targets should be divided into two parts. One of these groups would include the category of "important other targets" and the other group "other targets". That would facilitate the prioritization of targets for the destruction by fire.

The important other targets are the ones which can have an impact on the own troops soon, that is why their destruction is desirable as soon as possible. Such are the enemy's near reserves, march columns, heavy means of fire in an open position, groupings manoeuvring close to own troops, helicopters on landing ground, armour-piercing equipment, etc. Based on the tactical situation, some of the important other targets may be re-qualified as high value or high pay-off targets (HPTs). Similarly, targets having been assigned to the group of high value targets can be re-assigned to a lower priority group of targets. For example, if no air strikes are planned to be conducted in one of the defence phases of defence combat, then anti-aircraft artillery means will not be high value or high pay-off targets. On the other hand, in the course of counterattack by own forces, armour-piercing missiles will be high value or high pay-off targets.

Other targets are: those that have (or may have) an impact on own troops but cannot have decisive effects soon, and they can be destroyed by other equipment (e.g. all-arms), and/or destroying them soon is not the most important task. These include e.g.: personnel and means of fire in an open position. 
False targets include those own troop elements whose location has been specified by graphic intersection and reported by mistake, as well as the dummy and deceptive enemy targets.

During assessment, the target coordinates shall be subjected to a safety filtering.

This means that the software compares the target coordinates with the coordinates of own troop elements, and in case they match (there is no more than $200 \mathrm{~m}$ difference between the $\mathrm{x} y$ coordinates of the target and the own troop element), the software will indicate that and highlight these targets, grouping them separately as "False targets", and prompt to delete them. The front edge is also regarded as an own troop element (it is included in the data base), that is why the software will block the targets at the front edge, those whose location has been specified by graphic intersection and reported by mistake. Both groups of false targets can be printed in form of a chart.

In my opinion, this safety analysis and assessment phase is very important. I have directly experienced this problem at a corps-level intelligence exercise, where, during direct engagement with the enemy, our own reconnaissance sub-unit (artillery observation post) specified the location of own forward observation posts and reported them as targets several times, which we could discover through conventional data processing.

In the next phase of analysis and assessment work, the value of the targets (intelligence information) is defined, that is: the target data will be compared with the previously reported target data.

Single targets with single targets, single targets with groups of targets, and groups of targets with groups of targets will be compared to nature, type, coordinates, accuracy indicator of location (accurate or inaccurate), reliability indicator of the intelligence source, and the value of the intelligence information, among targets located in a circle with a certain (default: $200 \mathrm{~m}$ ) radius.

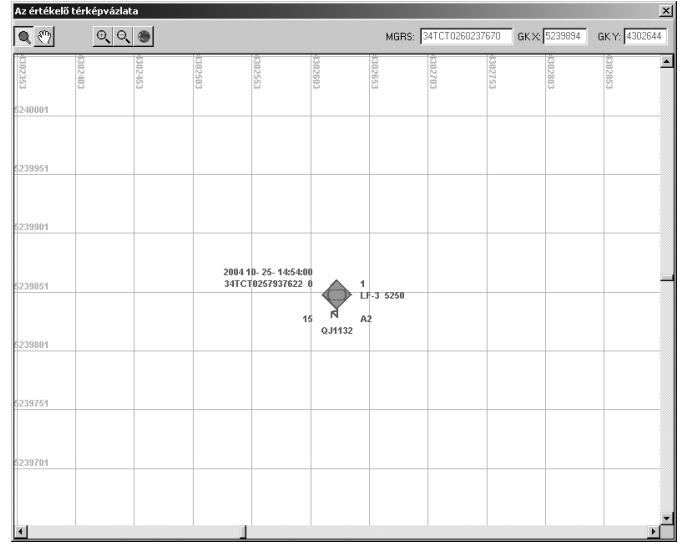

Figure 5. Analysis and assessment of targets on a digital map (own resources) 
The requirements of the analysis and evaluation work:

- the software shall be able to investigate targets detected within a specified time period (e.g. 1 hour);

- every previously reported target located within a circle with $200 \mathrm{~m}$ radius from the currently reported target shall be displayed, indicating also the distance. These targets shall also be displayed on the monitor with the standard symbols, the most recent one in a different colour — for distinction - (Fig. 10).

- if there is less than $75 \mathrm{~m}$ distance between the coordinates of one or more single targets of the same type, they shall be considered as one target, and the no. and coordinates of the target shall be those of the target located by the more accurate means. The other target(s) shall be invalidated and allocated to the data base of invalid targets.

- if the graphic intersection has been carried out by assets of the same type, and the difference is less than $75 \mathrm{~m}$, a mean coordinate $(\mathrm{E}, \mathrm{N})$ value shall be taken. The no. of the new target will be the no. of the target first reported.

- if the coordinates of a single target are compared with the coordinates of the centre of a group of targets, and the distance of $\mathrm{x}$ and $\mathrm{y}$ is less than $200 \mathrm{~m}$, the single target will be considered as part (sub-object) of the group of targets (confirmation of group of targets);

- if a single target is compared by coordinates with one or more targets of different or same nature, e.g. a tank, an armoured personnel carrier, a machine gun, an armourpiercing gun, etc., and the difference between the coordinates (E, N) is less than 400 $\mathrm{m}$, it shall be considered as part of a new group of targets.

- the new group of targets will have a new target no. (the software will issue a target no. from the target no. file), and the components (single targets) of the group of targets will also be recorded (stored) as sub-objects. The coordinates of the group of targets will be the mean value of the $\mathrm{E}$ and $\mathrm{N}$ coordinates of the single target components, its height will be the mean height values of the single target components. The software also calculates the width and depth of the generated group of targets, - in accordance with professional requirements - perpendicular to the basic direction.

- if the coordinates of a group of targets are compared with those of another group of targets, and the difference between the coordinates is less than $75 \mathrm{~m}$, then it is the confirmation of one group of targets. The no. and coordinates of the group of targets shall be those of the target located by the more accurate means. If the graphic intersection has been done by means having similar accuracy, a mean coordinate value shall be taken, and the no. of the new target will be the no. of the target first reported.

As such, groups of targets can be created during assessment:

1. Based on the report from the original intelligence source - an original group of targets;

2. If the original group of targets has been confirmed by separate reports on single targets - a group of targets confirmed through single target(s);

3. Creation of a group on the basis of single targets through analysis - new group of targets;

4. Confirmation of a group of targets by another group of targets. 
The assessed targets will be displayed on the analyser's digital map with NATO standard symbols (Fig. 10).

Assessment of the value of the target (intelligence information):

- The software will consider the targets (intelligence information) as confirmed by other sources if:

- " 1 ” is reported in column 17 of the "Intelligence Log” for the accuracy indicator of the information,

- two or more targets of the same nature and with a difference between the coordinates (x, y) of less than $75 \mathrm{~m}$, reported with information accuracy indicators "obviously correct” (“2”), “probably correct” (“3”), "highly doubtful” (“4”), “unlikely” (“5”), or "verity cannot be assessed” (“6”), - "not confirmed by other sources”, shall be considered as one target and will be rated as "confirmed by other sources” (“1”). The no. and coordinates of the new target shall be those of the target located by more accurate means. If the graphic intersections have been done by similar means, the no. of the new target will be the no. of the target first reported, and a mean coordinate value shall be taken.

- if the nature and coordinates (E, N) of a target "not confirmed by other sources” ("2", "3”, “4”, "5”, “6”) are the same (within $75 \mathrm{~m}$ ) as those of the target "confirmed by other sources" (" 1 ”), then the data of the target "confirmed by other sources" (" 1 ”) shall apply;

The software groups the targets "confirmed by other sources" also by priority, group of targets, and size: The targets are sorted in a chart, which can also be printed.

The evaluated targets are fed back to the data base of expected and detected targets, where they will be summarized by type and nature (Figure 2).

The software moves the reported dummy targets to a separate group (file) and displays them on the digital map with their NATO standard symbols.

By size of target, there are:

- groups of targets,

- single targets.

The targets "confirmed by other sources" need additional intelligence based on the cumulative assessment of their priority (threat), timeliness of intelligence data, and other aspects, usually immediately before destroying them, and this intelligence is to be carried out by the reconnaissance sub-unit that is going to support fire.

The software carries out the necessary visibility estimation regarding the target for the positions of all reconnaissance assets, taking into account their intelligence/reconnaissance capabilities - which can also be displayed on the digital map - and proposes what reconnaissance assets should be involved in additional intelligence. The polar coordinates and elevation of the target viewed from the position of the chosen reconnaissance asset will be displayed.

After destroying the target, the data bases "Amount of expectable and detected targets" and "List of destroyed targets" will be updated, and the symbol of destroyed target will show up on the digital map.

The reconnaissance sub-unit supporting fire will report data consistent with NATO requirements in column 19 of the "Intelligence Log”, as follows:

- the extent of destruction: 
FURJÁN Attila: Analysis and assessment of targets on a digital map...

- annihilation,

- neutralization,

- suppression,

- jamming;

- the no. and description (name) of destroyed objects;

- whether they can continue the activities: yes or no;

- the extent of destruction of the type of target (\%).

These data are recorded in the data base.

The target is to be considered as "not confirmed by other sources" if:

- nothing, or any of the following has been reported in column 17 of the "Intelligence Log”:

- obviously correct (“2”)

- probably correct (“3”),

- highly doubtful (“4”),

- unlikely (“5”),

- verity cannot be assessed (“6”),

or if:

- a target "confirmed by other sources" (“ 1 ”) and a deceptive ("Mt”) target have the same nature, and the difference between their coordinates (E, N) is less than $75 \mathrm{~m}$. (In that case "verity cannot be assessed" (6))

The targets "not confirmed by other sources" are allocated to a separate group, similarly to the confirmed targets. Every unconfirmed target needs additional intelligence, since the data about them do not allow their immediate destruction. These targets are sorted in a chart, which can also be printed.

The software proposes what reconnaissance assets should be involved in additional intelligence based on the visibility examination and the reconnaissance capabilities of reconnaissance assets.

After the additional intelligence, the targets can be allocated to different groups depending on the assessment and processing, such as to the:

- group of targets “confirmed by other sources” (“1”);

- group of targets classified as "verity cannot be assessed” (“6”),

- group of deceptive (“Mt”) targets.

The targets, owing to their high manoeuvrability, change their positions quickly in the course of intelligence. Therefore, the reconnaissance personnel are obliged to report to the data processing centre if targets leave the previously reported positions. In that case, the target will be moved from the actual data base to the group of "invalid targets". This group of targets can be printed in form of a chart.

The digital map also allows target planning using the software. The planned targets will be drawn on the analyser's digital map with NATO standard symbols.

A reconnaissance-observation source has to be assigned to the planned targets. This is easy by the visibility examination carried out by the software. The software will examine which reconnaissance source (included in the data base) has sight of the target, taking into account the reconnaissance capabilities. It will display the visibility list, after which the analyser-evaluator shall choose the intelligence sources. When they are chosen, the software 
displays the polar coordinates and elevation of the target viewed from the position of the reconnaissance asset. The data will be transmitted to the designated reconnaissance asset through digital transmission or conventional telecommunications, and, using those data, they will carry out aiming at the target in the field. The data about the planned target, together with the data of the chosen intelligence source will be recorded in the chart "planned and real targets”, and can be printed.

During the analysis and assessment work, all intelligence data about the target can be recalled — by selecting the standard symbol of the target — on the digital map.

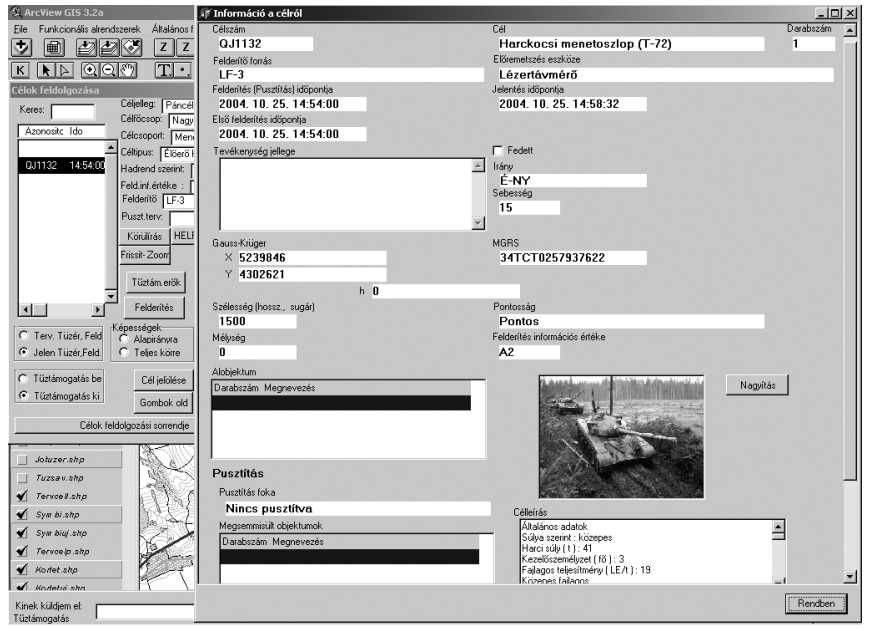

Figure 6. Recalling data about detected targets from the data base (own resources)

Drawing conclusions is the last step of information analysis. During drawing conclusions, we will find out what significance the intelligence data has in the combat area. They might be helpful for the anticipation of the future activities of the enemy.

\section{Usage, distribution}

The last step of the intelligence process is usage or distribution. The information shall immediately be made available to the user. Access to the intelligence data shall be granted to all concerned command levels and work groups. In my opinion, the intelligence data processing group and the fire support section (who allocate targets among forces and fire means) shall work in close cooperation, and they should be located next to each other, in accordance with NATO principles.

The examination of "important other" and "other" targets is carried out by the software analogously.

Basic requirements for the digital map software:

- display the intelligence data (targets) assessed by the computer with NATO standard symbols on the map; 
- during the analysis of the displayed targets, all of their intelligence data shall be recallable (e.g. time of reconnaissance, nature of target, UTM (MGRS) coordinates, height, width (length), depth, description of the intelligence source, accuracy and reliability indicators, combat properties, parameters, and imagery of the target, the value of the intelligence information);

- a distinctive symbolization of destroyed targets, and all the information regarding the target and the destruction shall be recallable (e.g. by which sub-unit and when it was destroyed, the result of destruction — damage in \%, no. and description/name of destroyed objects);

- display own reconnaissance forces and assets with the standard symbols;

- drawing up the reconnaissance capabilities for all positions of reconnaissance assets;

- visibility examination and drawing up the visible (and invisible) area for both the own and enemy reconnaissance assets and targets (this is of great help in planning intelligence and proper and fast designation of the location of reconnaissance assets);

- drawing up the Brigade combat area (Division defence zone) with NATO standard symbols;

- drawing up the front zone with standard symbols using the reports by reconnaissance sub-units including polar coordinates, as well as by putting directly into the digital map;

The front edge can be quickly and properly modified on the digital map during combat, and the map information can be immediately forwarded to addressees via digital data transmission.

\section{Conclusion}

The experience of the exercises conducted with "HUTOPCCIS" has been promising and demonstrates that, taking conventional planning and combat command and control into account, computer-aided tactical control can provide faster and more reliable information to headquarters and staff. The conventional telecommunications methods and tactical control, and the information flowing through an IT system complement and reinforce each other, making command and control more reliable.

\section{References}

[1] Assessment of intelligence data. Budapest: General Staff Euro-Atlantic Integration Work Group, 1996.

[2] Voennaja Musl. 1994. 1. Issue

[3] Intelligence Doctrine. Budapest: General Staff Euro-Atlantic Integration Work Group, 1996. 